\title{
THE CARDIOGRAM IN VENTRICULAR ANEURYSM FOLLOWING CARDIAC INFARCTION
}

\author{
BY \\ TERENCE EAST and SAMUEL ORAM \\ From the Cardiological Department, King's College Hospital \\ Received August 4, 195 I
}

The most reliable single aid in the diagnosis of ventricular aneurysm is fluoroscopy and the value and difficulties of this method of diagnosis have been fully dealt with by Parkinson et al. (1938). If one accepts the definition of Matthew Baillie (1793), which runs " This disease consists in a part of it (the heart) being dilated into a pouch which is commonly more or less filled with coagulated blood," those cases that show merely an excavation of the inner surface of the ventricle should be excluded: they would not be diagnosed by fluoroscopy. There is no doubt that ventricular aneurysm can be present and yet careful rotation of the patient at fluoroscopy may show only an enlarged left ventricle. On the other hand, what is considered to be radiological evidence of ventricular aneurysm may be found to have been misleading (see Case 7) and even when radiological signs of aneurysm appear to be present they may be only transient (see Case 4). The radiological diagnosis of posterior cardiac aneurysm is particularly difficult, and we have been able to find very few convincing illustrations.

There is thus a need for some other aid in the diagnosis of ventricular aneurysm following cardiac infarction and according to Samet et al. (1950) the electrokymogram may soon prove of value. In the meantime, there is no doubt that a striking divergence of opinion exists concerning the value of the cardiogram in the diagnosis of ventricular aneurysm, as recently revealed by Rosenberg and Messinger (1949).

Naturally we wondered whether some of the cardiographic appearances said to be suggestive or typical of ventricular aneurysm could not in fact have been produced by large transmural infarcts not necessarily accompanied by any bulging. After all, as pointed out by Gross and Schwedel (1941), the resilience of the resulting scar depends on many factors, such as the size of the area infarcted, the site of the infarct, and the presence or absence of intramural thrombus, calcification, or possibly pericardial adhesions. Perhaps the intraventricular pressure may count as well. We feel that probably the most important cause is the extent to which a large infarct has involved the thickness of the ventricular wall and there is some cardiographic support for this opinion in the form of QS waves. In order to decide whether these so-called typical appearances of aneurysm were due merely to the presence of a large transmural infarct, with or without aneurysm formation, we have compared two distinct types of case. The first group consisted of patients in whom there was evidence of cardiac aneurysm either at autopsy or radiologically: the second, of patients in whom there was a large transmural infarct of the left ventricle and yet at autopsy it was noted that no aneurysm was present. We have also included some details of a much smaller group where no autopsy was obtained and the radiological appearance, coupled with the clinical findings, made the diagnosis of ventricular aneurysm likely but not certain. A point of some importance that we would wish to emphasize, is that search for ventricular aneurysm must be made when the material is fresh. It is surprising how shrinkage due to formalin can reduce the dimensions of an aneurysmal 
bulge or obliterate it altogether unless the clot is removed and the cavity packed with cotton wool before fixation.

\section{MATERIAL AND RESUltS}

We divided our material, consisting of 42 cases, into three groups (see Table). First, those in which a diagnosis of ventricular aneurysm was considered to be certain: there were 27 cases; in 11 autopsy confirmed the presence of ventricular aneurysm, and in the remaining 16 the radiological appearance was beyond doubt (Fig. 1). Secondly, a group of 12 cases in which at autopsy a large transmural infarct was present but gave rise to no ventricular aneurysm. Thirdly, 3 cases where the presence of ventricular aneurysm was suspected clinically and radiologically but we could not be certain that one was present and no autopsy was obtained.

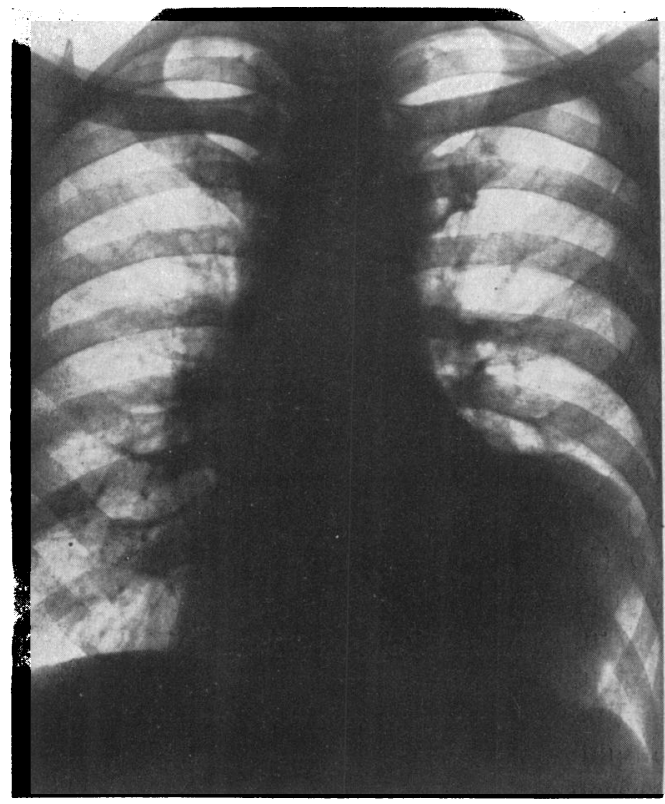

A

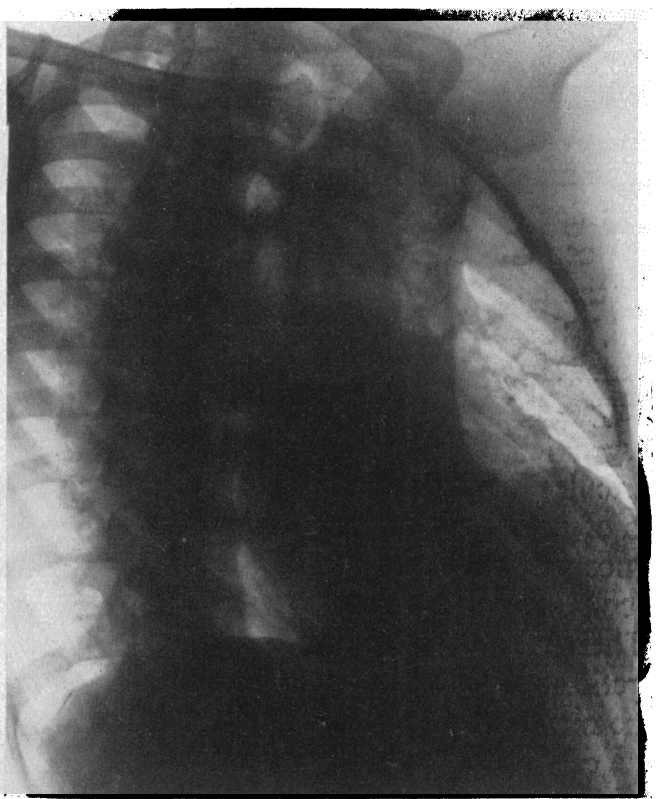

B

FIG. 1.-Case 1. (A) Antero-posterior view. (B) Right anterior oblique view. Large ventricular aneurysm present.

Of the group known to have a ventricular aneurysm, it was anterior in 24 and postero-apical in 3 cases. No case of a purely posterior infarct leading to aneurysm was encountered for the $T$ waves in the leads from the left of the præcordium were also inverted.

Of the 12 cases with large infarcts without aneurysm 9 were anterior, 2 were both anterior and posterior, and 1 was entirely posterior. All of the three suspected but unconfirmed cases of aneurysm were thought to be anterior.

\section{Ventricular ANEURYSM}

On analysing the curves obtained from the 24 examples of cardiac aneurysm arising from anterior infarction, it at once became obvious that a certain pattern was common. Not only is the pattern frequently seen, but once it appears it persists. It consists of the following features.

In the Chest Leads

(1) The presence of a QS wave ( $R$ being absent) in at least one (V1 excluded) of the unipolar chest leads. 
TABLE I

SUMMARY OF MATERIAL

\begin{tabular}{|c|c|c|c|c|c|c|c|}
\hline \multirow[b]{2}{*}{ Type of Case } & \multirow[b]{2}{*}{ Total } & \multirow[b]{2}{*}{ Autopsy } & \multirow[b]{2}{*}{ Radiology } & \multicolumn{4}{|c|}{ Site of Cardiac Aneurysm or Infarct } \\
\hline & & & & Anterior & Posterior & $\begin{array}{l}\text { Anterior } \\
\text { and } \\
\text { posterior }\end{array}$ & $\begin{array}{l}\text { Postero- } \\
\text { apical }\end{array}$ \\
\hline \multirow{2}{*}{ Proven aneurysm } & \multirow{2}{*}{27} & 11 & & $10^{*}$ & & & 1 \\
\hline & & & 16 & 14 & & & 2 \\
\hline Suspected aneurysm & 3 & & 3 & 3 & & & \\
\hline $\begin{array}{l}\text { Infarct without } \\
\text { aneurysm }\end{array}$ & 12 & 12 & & 9 & 1 & 2 & \\
\hline
\end{tabular}

* One of these cases had two anterior infarcts, only one of which had led to aneurysm formation. In another anterior case the infarct had also resulted in an aneurysm of the ventricular septum.

(2) Elevation of the RS-T segment in the unipolar chest leads, this being variable in occurrence and duration.

(3) Widespread inversion of the T wave, or its incorporation in the elevated RS-T segment, in at least three of the unipolar chest leads.

In the Limb Leads

(4) A Q wave in VL, and therefore in standard lead I.

(5) Inversion of the T wave in VL, and consequently in standard lead I.

(6) A deep S wave in VF, $5 \mathrm{~mm}$. or more in depth, and consequently in standard leads II and III.

Four of these six features were constantly present in no fewer than 18 of the 24 cases, namely (1) one or more QS waves, (3) widespread inversion of the T wave in the chest leads, (5) inversion of the T in VL, and (6) a deep S wave in VF (Fig. 2). The two inconstant features were a $Q$ wave in VL which was present in 15 of the 18 cases; and elevation of the RS-T segment which occurred in 12 of the 18 cases, but in 3 of the 12 it was of short duration only. We consider that undue emphasis has been placed on the presence and persistence of RS-T segment elevation in the diagnosis of cardiac aneurysm. It is often absent in cardiac aneurysm, and can be present and persist in large antero-lateral infarcts in the absence of aneurysm formation.

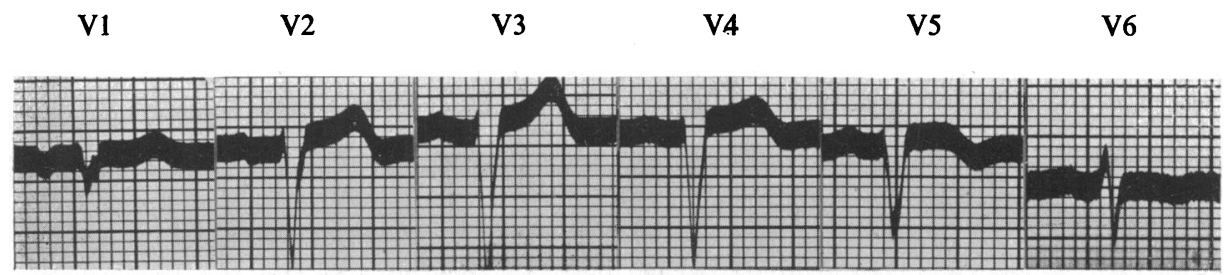

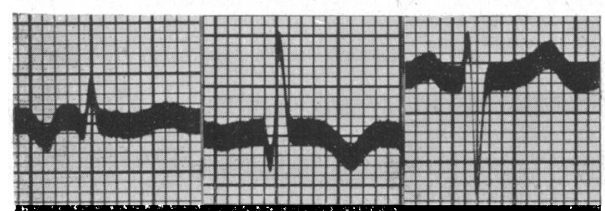

VR

VL

VF

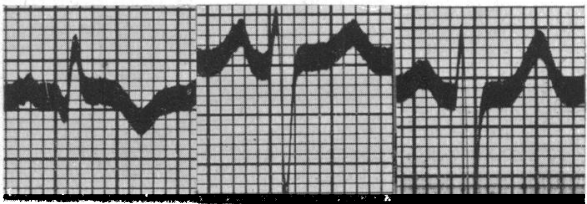

I

II

III

FIG. 2.-Case 1. The four features of ventricular aneurysm are the presence of a $Q S$ wave in at least one of the unipolar chest leads, inversion of the $T$ wave in some of the chest leads, inversion of the $T$ wave in leads VL and I, and a deep $S$ wave in leads VF, II, and III. In addition a $Q$ wave is present in leads VL and $I$. 
Of the remaining 6 cases out of the 24, four had a QS wave in standard lead I, and two of these showed a deep S in VF, and thus in standard leads II and III, with extensive inversion of the $T$ wave in the chest leads. The other two with QS waves in standard lead I had no such deep $S$ waves in VF, II, or III, but had inversion of the T waves in the chest leads. . The fifth case resembled the 18 "typical" cases with a $Q$ wave and an inverted $T$ wave in leads VL and I and extensive inversion in the chest leads, except that there was no $S$ wave in leads VF, II, or III. This is the only example we have been able to find of such a curve in the presence of anterior ventricular aneurysm. The electrocardiogram and radiological appearance are illustrated in Fig. 3 and 4 (Case 2) and will be commented upon later. The sixth case had all the "typical" cardiographic features except that the $\mathrm{S}$ wave in standard lead II was absent.

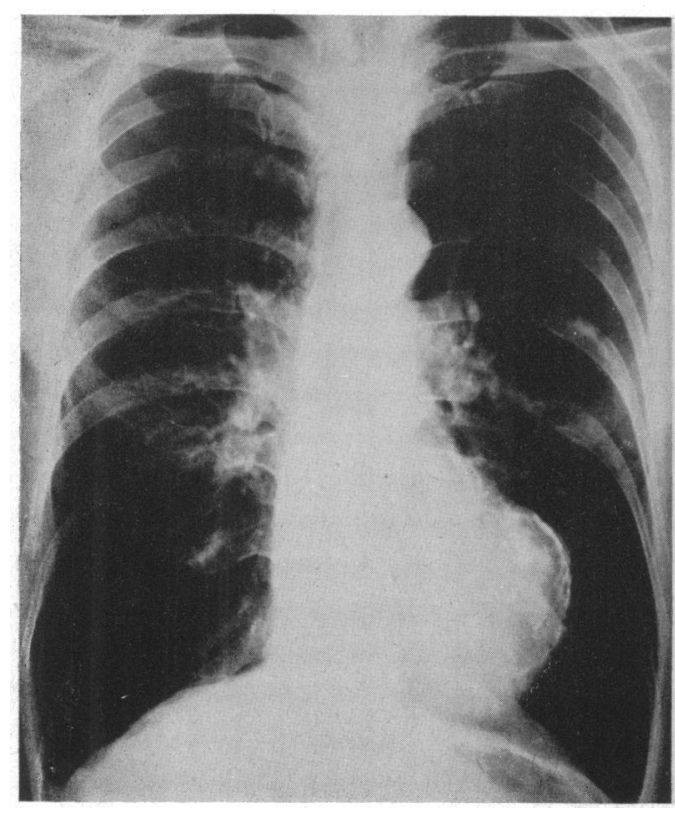

A

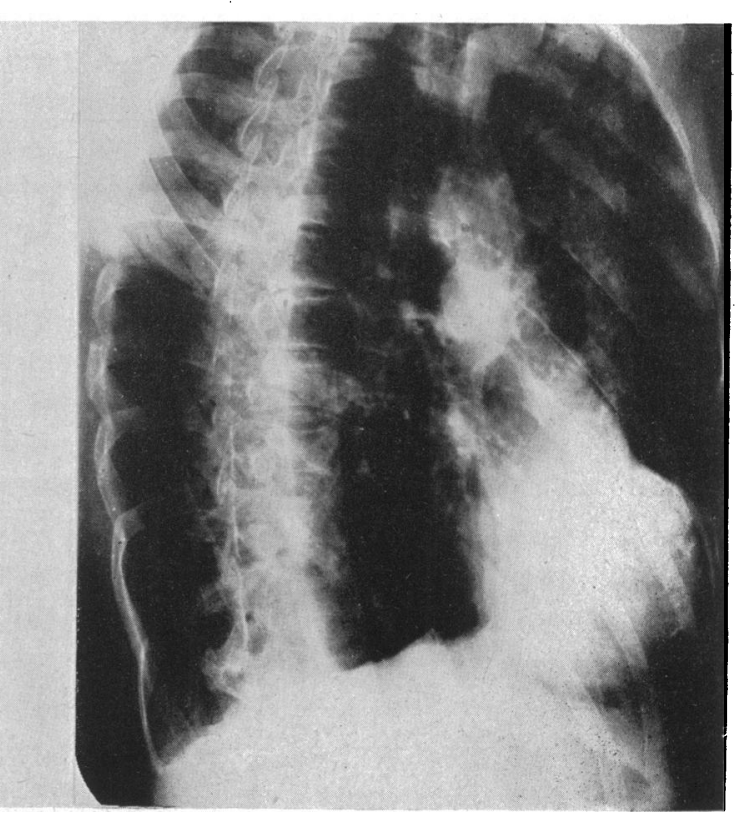

B

FIG. 3.-Case 2. (A) Antero-posterior view. (B) Right anterior oblique view. Calcified aneurysm of left ventricle.

The curves from the three cases of postero-apical infarction with posterior aneurysm did not reveal any features other than those to be expected from a transmural infarct, except that, as already mentioned, all three were postero-apical and not just posterior. All three had a QS wave in VF. None of them showed well developed elevation of the RS-T segment; in two of them we took osophageal leads and no such elevation was seen in those leads either, nor was any further clue as to the presence of aneurysm obtained.

Of the three doubtful cases of aneurysm two had the six typical features mentioned above, and, although radiologically we could not be certain of an aneurysm, we felt it almost certainly was present in the two with that type of cardiogram. The other doubtful case was probably not one of cardiac aneurysm, as the cardiogram was within normal limits in spite of the suggestive radiological appearance (see Case 7, Fig. 11 and 12).

\section{INFARCTION WITHOUT ANEURYSM}

Of the 12 examples of large transmural infarct without aneurysm, nine were anterior, one was posterior, and two were both anterior and posterior. 
When we considered the curves obtained from the nine extensive anterior infarcts without aneurysm formation, we were surprised to see that in none of them did the curves show all the four criteria mentioned previously, namely (1) the presence of one or more QS waves, (3) widespread inversion of the $T$ wave in the chest leads, (5) inversion of the T wave in VL, and (6) a deep S wave in VF. In one case only (Case 9, Fig. 14) did the cardiographic appearances resemble these criteria, and that was an example where at autopsy in addition to a large anterior infarct a recent posterior infarct was also present. Of the nine anterior cases, eight showed the usual changes in the chest leads of anterior cardiac infarction but without the other criteria of aneurysm in the unipolar limb leads that we have described; and in the other case the appearance was obscured by auricular flutter with 1:2 ventricular response. In two of the eight cases, however, there was a deep $\mathrm{S}$ in

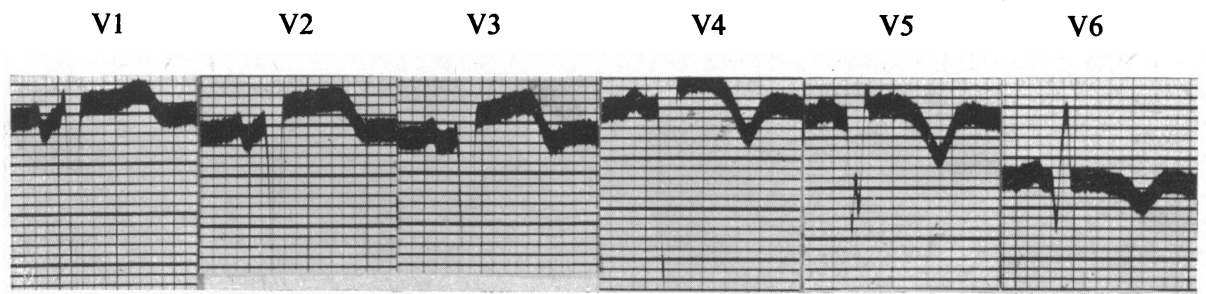

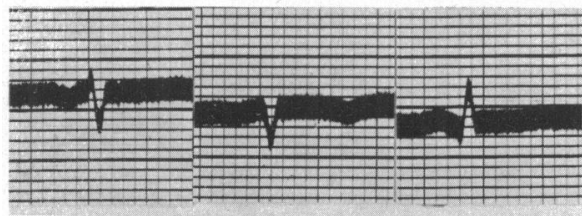

VR

VL

$\mathrm{VF}$

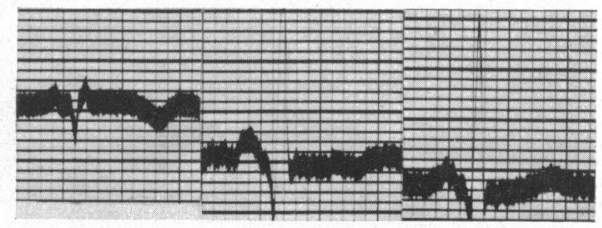

I

II

III

Fig. 4.-Case 2. In spite of the presence of a ventricular aneurysm (Fig. 3) there is no $\mathrm{S}$ wave in leads VF, II or III.

VF, and so in standard leads II and III; but in one of these the chest lead T waves were not inverted, and in the other no chest leads were taken.

The biggest series of large antero-lateral infarcts that has been fully explored cardiographically and correlated at autopsy is that of Myers et al. (1948). They give details of 57 such cases and from study of their illustrations, provided recent infarction is excluded, we were unable to find an example showing all of the six cardiographic features we have described, except when a cardiac aneurysm was present. Of their four examples of actual cardiac aneurysm, three showed the appearance such as we have noted (their Fig. 15).

The example of large posterior infarct without aneurysm could not be distinguished cardiographically, even with osophageal leads, from the three that had a posterior aneurysm at autopsy, except that in the aneurysm cases the inversion of the $T$ wave had spread round to the front of the left ventricle whereas it had not done so in the posterior infarct case without aneurysm.

\section{Illustrative CASES}

Case 1. Male, aged 64. History of cardiac infarction with pain lasting four hours, development of a pericardial rub on the third day, and later appearance of left ventricular gallop rhythm. The cardiogram (Fig. 2) showed the six typical features of aneurysm that we have described. Although no autopsy was obtained there is no doubt from the X-ray appearance (Fig. 1) that a large ventricular aneurysm was present.

Case 2. Male, aged 60. This large ventricular aneurysm with calcification of its wall (Fig. 3) is one of the few examples where the S wave was absent in leads VF, II, and III (Fig. 4). In spite of the absence of pain in the chest for twenty-two years, considerable RS-T elevation persists in leads V2 to V5 and a QS wave is present also in those leads. We are grateful to Dr. G. A. Kiloh for details of this patient. 
Case 3. Male, aged 72. A large cardiac aneurysm at autopsy had caused such thinning of the left ventricular wall that on removing the clot from the aneurysm light could be seen through it. The cardiogram (Fig. 5) was unusual in that, in addition to the absence of a $Q$ wave in leads VL and $I$, no $R$ wave was present in VR, although according to Goldberger and Schwartz (1948) this would indicate the absence of ventricular aneurysm. The aneurysm had caused a peculiar elongation of the left ventricle (Fig. 6) as originally described by Hochrein (1932).

Case 4. Male, aged 55. This illustrates how the radiological appearances can be deceptively transient in the presence of cardiac aneurysm. On 16/8/50 an adhesion was present near the apex of the left ventricle (Fig. 7) and the cardiogram (Fig. 8) showed QS waves in leads V1 to V3, elevation of the RS-T segment in V1 to V4, inversion of the T wave in leads V3 to V6, as well as the QI, TI, SVF, SII and SIII appearance in

$\begin{array}{llllll}\text { V1 } & \text { V2 } & \text { V3 } & \text { V4 } & \text { V5 } & \text { V6 }\end{array}$

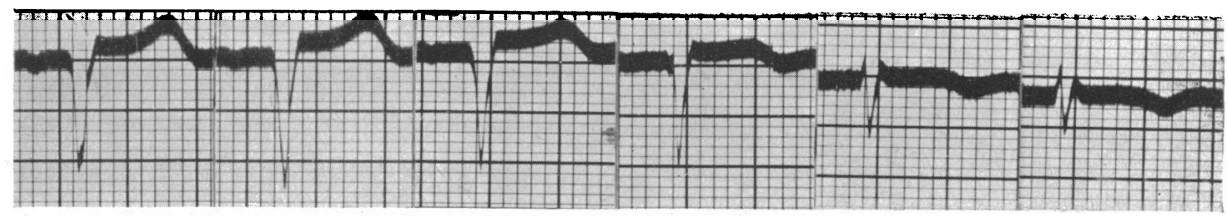

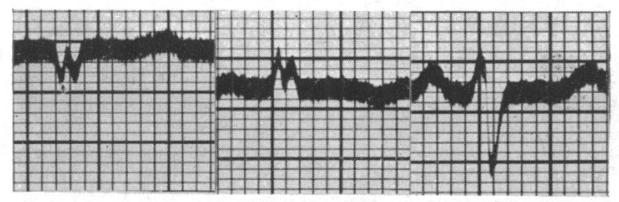

VR

VL

VF

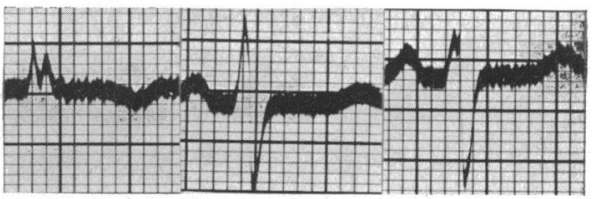

II

III

Fig. 5.-Case 3. The unusual features of this cardiogram of a ventricular aneurysm (Fig. 6 (A) and (B)) are the absence of an $R$ wave in lead VR and of a $Q$ wave in leads VL and $\mathrm{I}$.

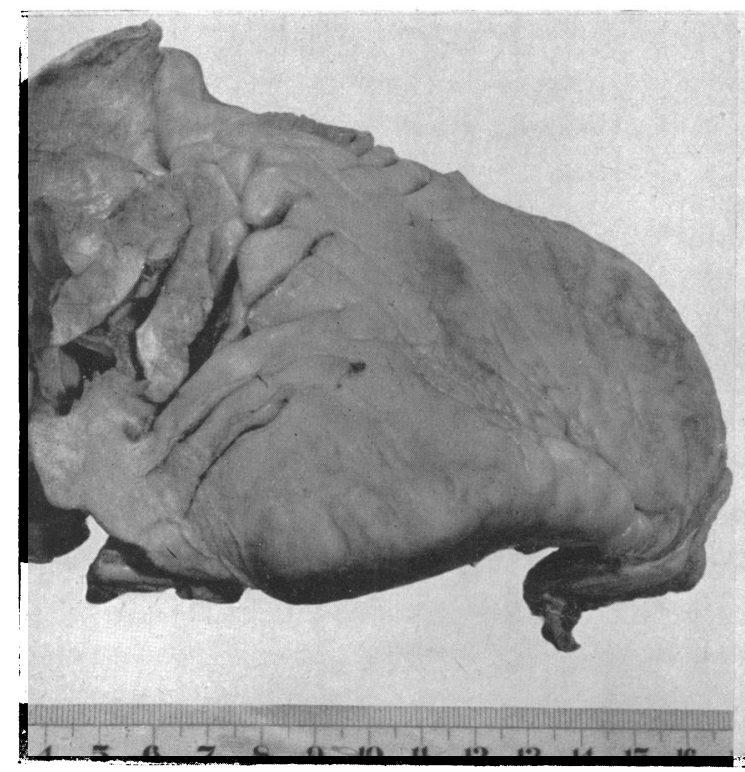

A

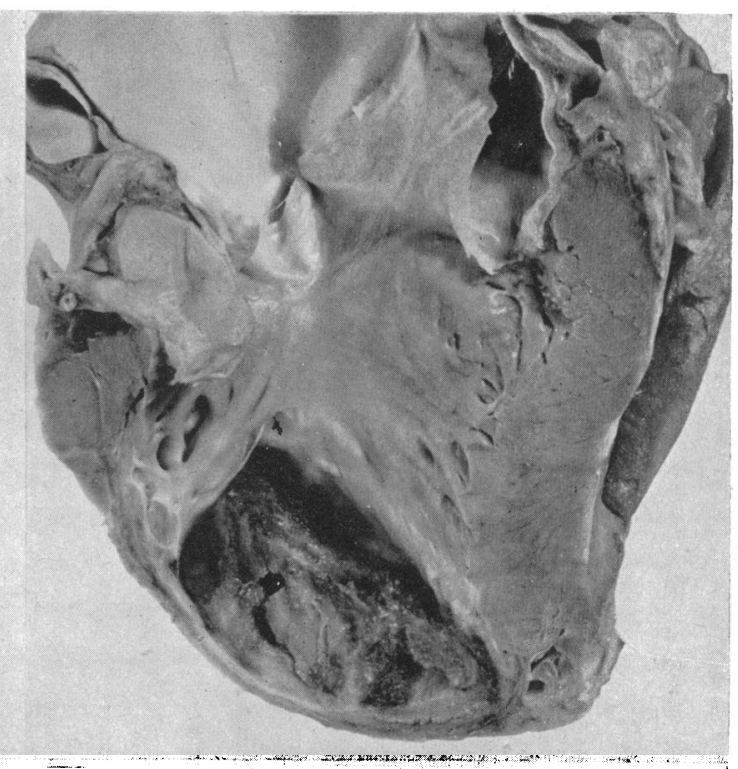

B

Fig. 6.-Case 3. (A) and (B) show elongation of the left ventricle resulting from ventricular aneurysm. 
the limb leads, all suggesting anterior aneurysm. However, by 2/11/50 the appearance at the apex had altered and the left ventricular contour was smooth in all positions at fluoroscopy (Fig. 7). We presume that the appearance of $16 / 8 / 50$ was due to an adhesion overlying the ventricular aneurysm which some time between that date and 2/11/50 had broken down. The patient had an apex beat suggestive of cardiac aneurysm in that two distinct and slightly asynchronous thrusts could be felt in the region of his apex a little outside the left mid-clavicular line.

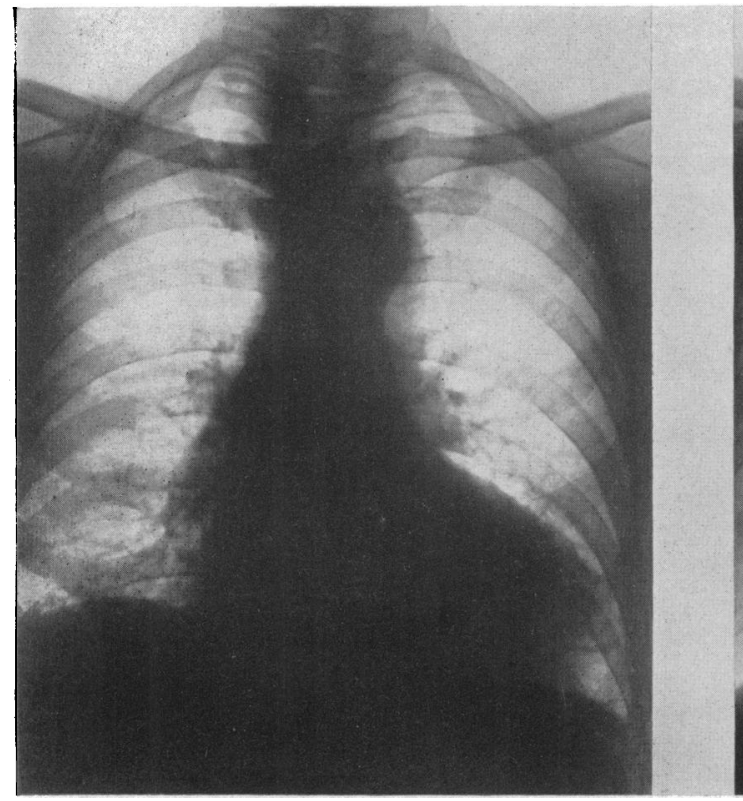

A

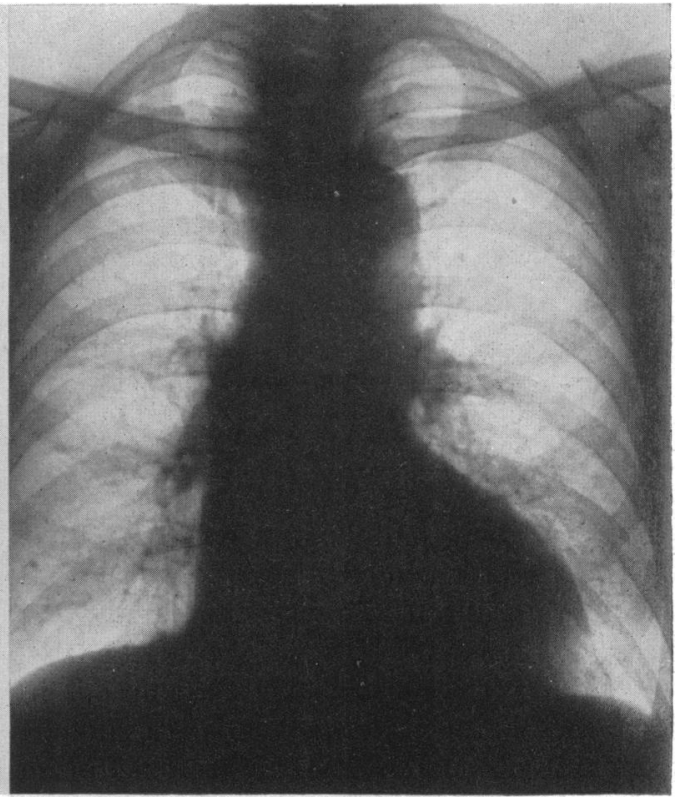

B

Fig. 7.-Case 4. (A) Antero-posterior view taken on 16/8/50. An adhesion is present near the apex of the left ventricle. (B) Same view taken on $2 / 11 / 50$. The left ventricular contour is smooth.

V1

V2

V3

V4

V5

V6
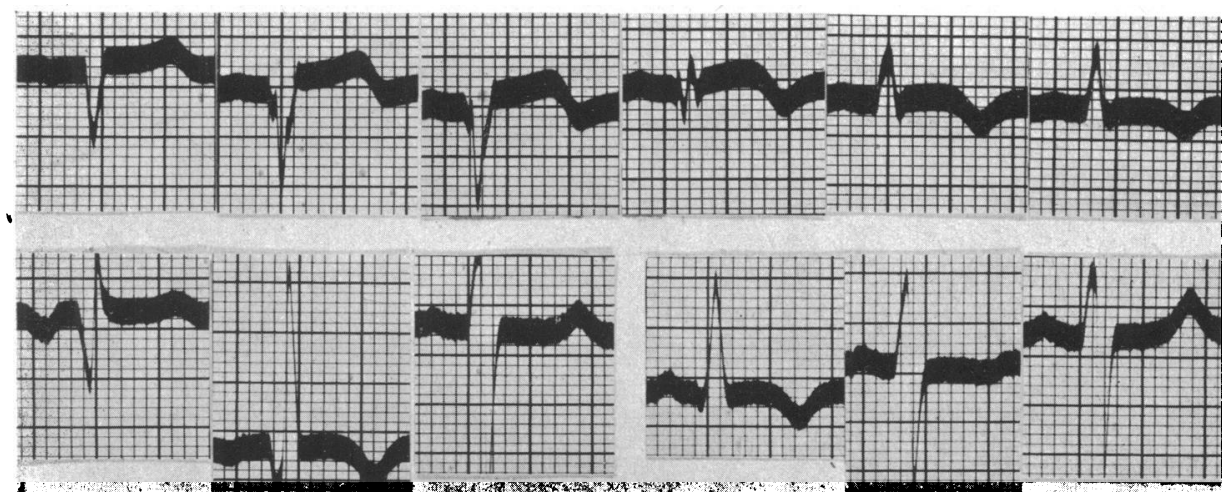

VR

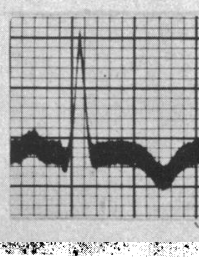

I

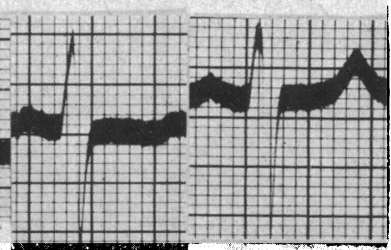

II
III

FIG. 8.-Case 4. Cardiogram of ventricular aneurysm in spite of the radiological appearance of Fig. 7 (B), namely one or more QS waves and widespread inversion of the T wave in the chest leads, inversion of $T$ in VL and I, and a deep $S$ wave in leads VF, II, and III. 
Case 5, male, aged 58, and Case 6, male aged 41. These two examples illustrate the persistence of considerable elevation of the RS-T segment which is sometimes found. In Case 5 the patient had experienced no cardiac pain for the previous two years and in the right oblique view a ventricular aneurysm was seen. In addition to the other cardiographic features of aneurysm there was considerable RS-T segment elevation in leads V2 to V5 (Fig. 9). Case 6 had had his infarct four and a half months prior to the cardiogram illustrated (Fig. 10) and at fluoroscopy on the same day as the cardiogram, a localized area of paradoxical pulsation was seen $1 \mathrm{~cm}$. above the apex of the heart. The record illustrates well the RS-T segment elevation which is seen in leads V2 to V5 and it is also a good example of the presence of QS waves in the chest leads - in this case in leads V1 to V5 inclusive. The other appearances associated with an aneurysm are also seen.

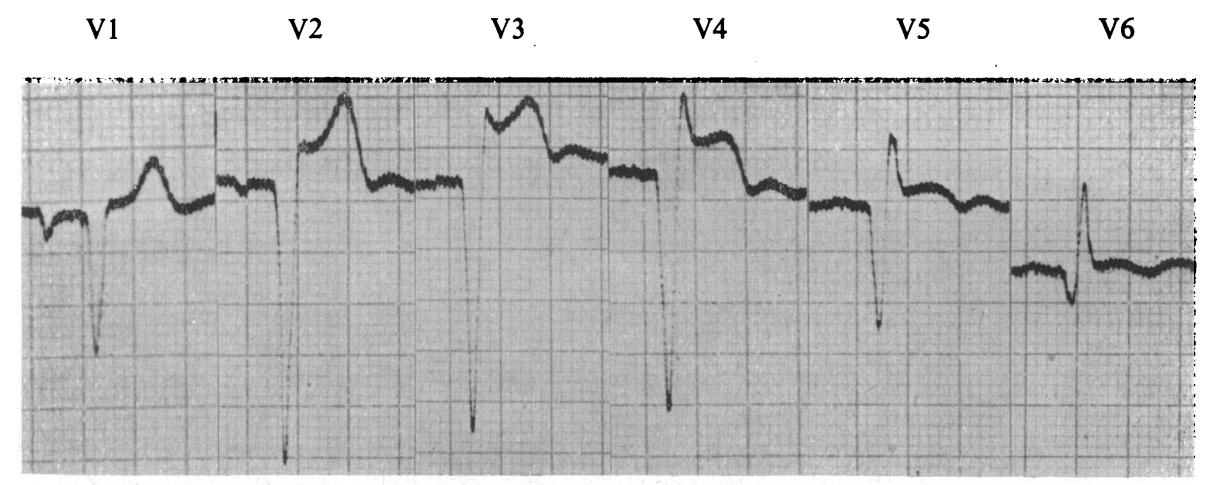

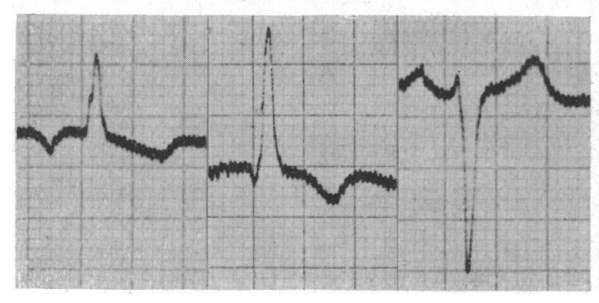

VR

$$
\text { VL }
$$

$$
\text { VF }
$$

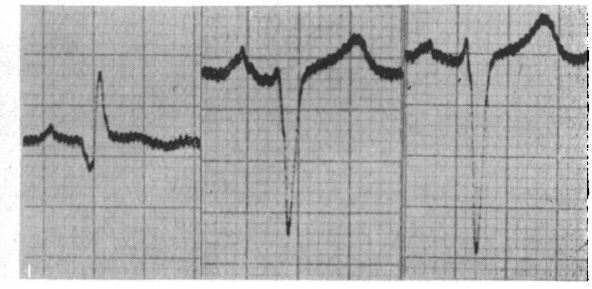

II

III

Fig. 9.-Case 5. In addition to the four commonest features of ventricular aneurysm (see text), and the presence of a $Q$ wave in leads $V L$ and $I$, considerable elevation of the RS-T segment is seen and has been present for two years.

Case 7. Male, aged 58. This is an example where the radiological evidence was again probably misleading (Fig. 11). The patient had severe sub-sternal pain in April, 1944, and clinically there was separate pulsation in two nearby areas near the left nipple. However, his cardiogram taken on the same day as Fig. 12 did not confirm the presence of an extensive infarct. A second twelve-lead cardiogram taken a year later showed no alteration. We therefore doubt whether the radiological appearance was that of ventricular aneurysm. Pericardial adhesions which distort the shadows of the left ventricular border are not uncommon in the absence of aneurysm (Schwedel, 1948).

Case 8. Male, aged 53. This patient is also one from our doubtful group. The cardiographic appearances were strongly suggestive of the presence of a ventricular aneurysm, namely the presence of a QS wave in lead V2 with T wave inversion in leads V2, V5, and V6, with a deep S wave in VF and thus in SII and SIII, together with a $Q$ wave and inverted $T$ wave in leads VL and I. Fluoroscopy did not reveal an aneurysm, and final diagnosis must await autopsy. As only lead V2 shows a QS wave (Fig. 13) it is possible that an aneurysm was present but was too small and too anterior to be demonstrated at fluoroscopy.

Case 9. Female, aged 62. In spite of the presence of an extensive anterior infarct with also a recent large posterior infarct high up in the septum, and the cardiographic appearances somewhat resembling those such as we found accompanying aneurysm, autopsy revealed no evidence of a local bulge. It will be seen (Fig. 14) that the T wave is inverted in leads V2 to V6 inclusive and in leads VL and standard lead I, and that 
V1

V2

V3

V4

V5

V6

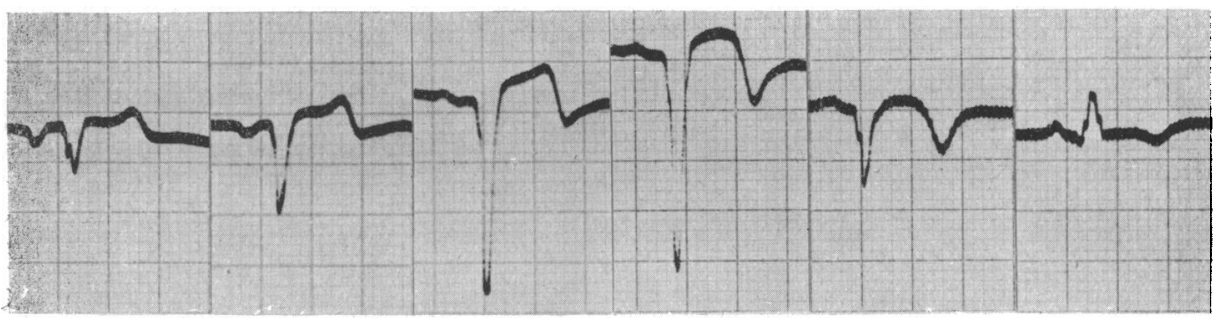

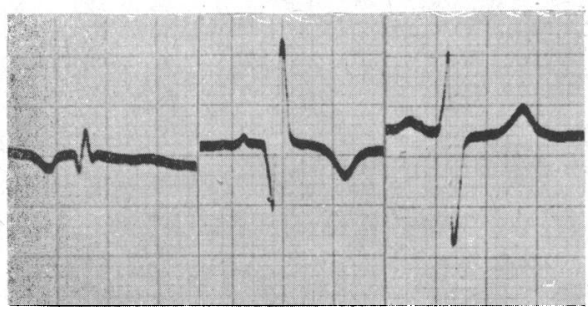

VR

VL

VF

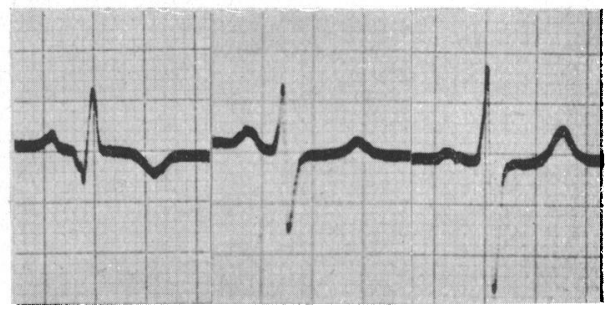

I

II

III

Fig. 10.-Case 6. An example of the presence of widespread QS waves and persistent elevation of the RS-T segment in the chest leads in ventricular aneurysm.

Fig. 11.-Case 7. Ventricular aneurysm was suspected because of a history of severe substernal pain, the presence of two separate impulses near the left nipple, and paradoxical pulsation of the left ventricular border. However, the cardiogram was not consistent with that diagnosis (Fig. 12).

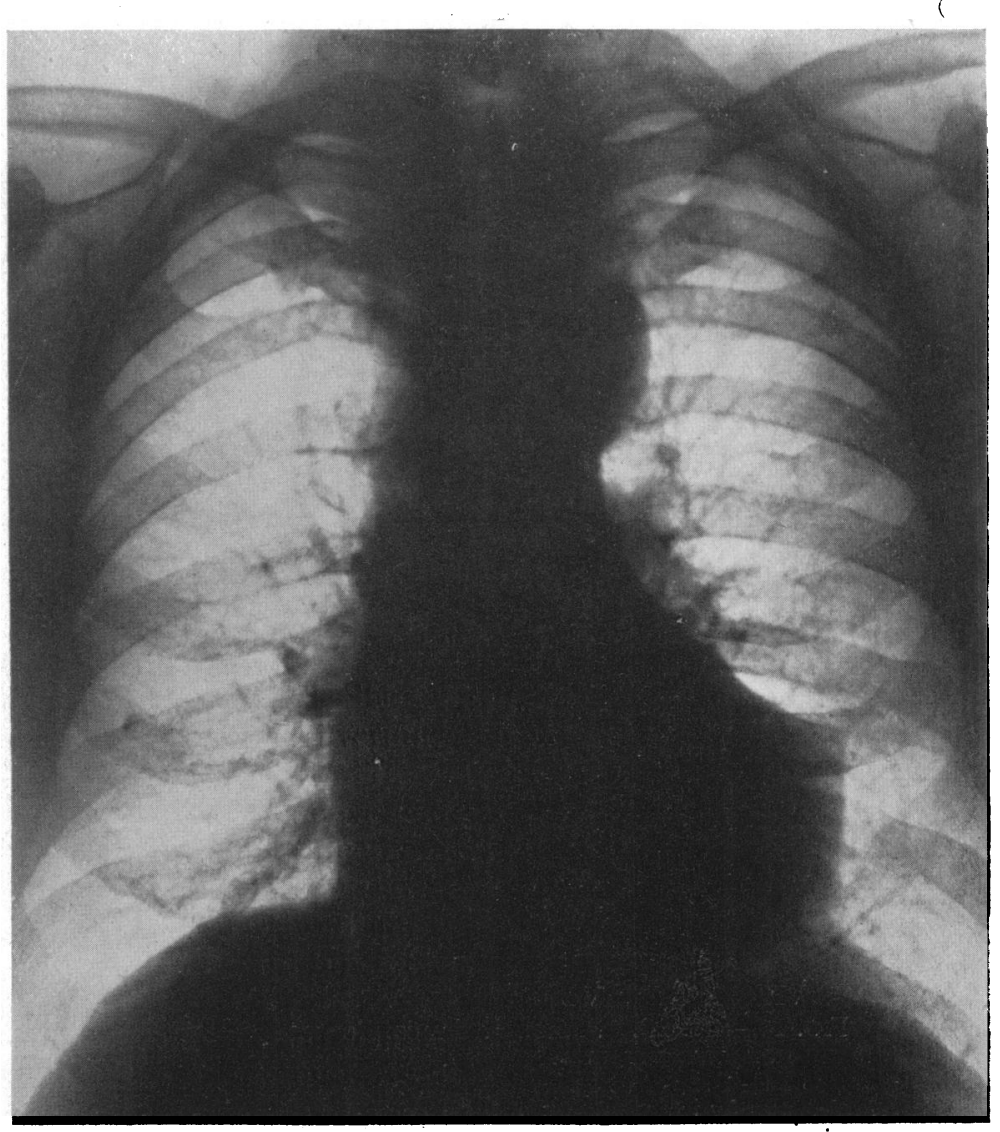




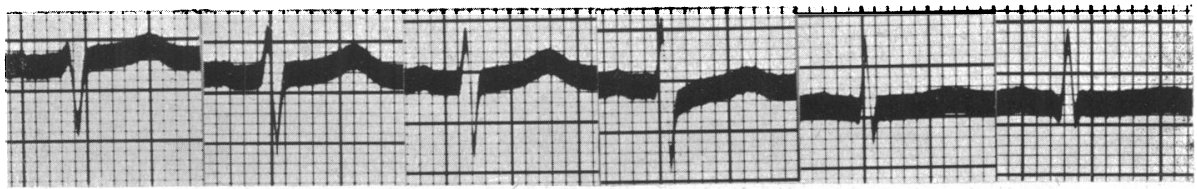

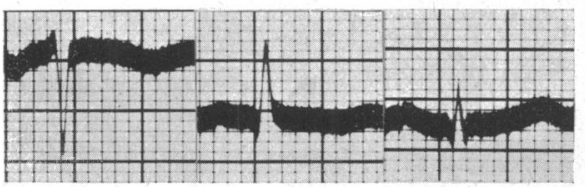

VR

VL

VF

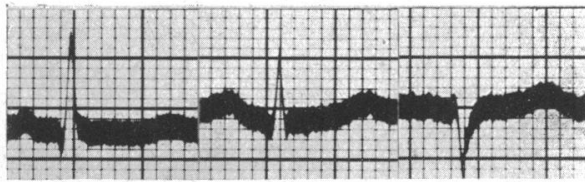

I

II

III

FIG. 12.-Case 7. Cardiogram is not typical of ventricular aneurysm although perhaps suggestive of some ischæmia of the left ventricle.

V1

V2

V3

V4

V5

V6

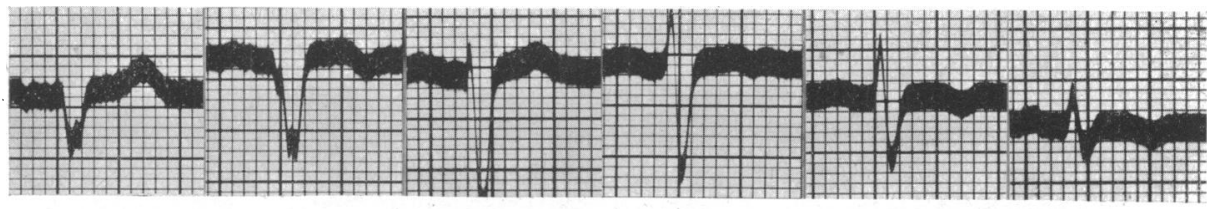

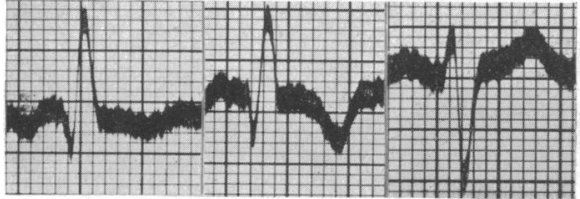

VR

VL

VF

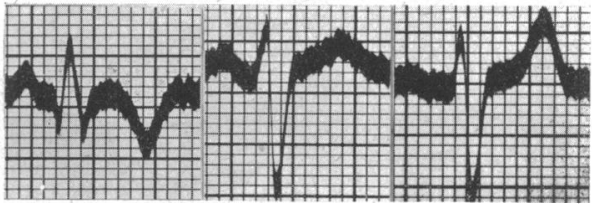

I

II

III

FIG. 13.-Case 8. Cardiogram showing all six features of ventricular aneurysm (see text) yet fluoroscopy failed to confirm its presence.

V1

V2

V3

V4

V5

V6
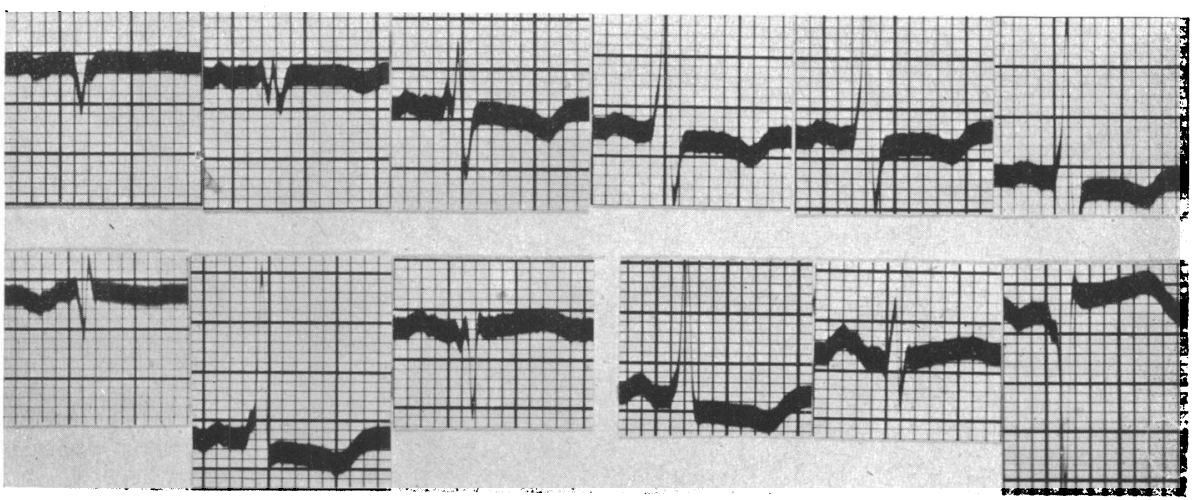

VR

VL

VF

I

II

III

Fig. 14.-Case 9. Although the cardiogram resembles that found in cardiac aneurysm none was present at autopsy. The asymmetrical inverted $T$ wave and depression of the RS-T segment in leads VL, $I$, and the chest leads suggest that left ventricular hypertrophy is contributing to the record. 
a deep S wave is present in leads VF and standard leads II and III. But the RS-T segment is depressed in lead I and elevated in lead III and we consider that at least some of this appearance is due to left ventricular hypertrophy (the heart weighed $507 \mathrm{~g}$.), and the deeply negative complex in lead VF and III almost certainly represents a QS wave due to the large recent posterior infarct in addition to the old anterior one.

\section{DisCUSSION}

Nordenfelt (1939) seems to have been the first to investigate the problem as to whether a ventricular aneurysm gave cardiographic changes peculiar to itself as opposed to those resulting from infarction without aneurysm. He stated that large aneurysms of the anterior wall of the left ventricle gave. a relatively low $R$ wave in lead I with a negative or isoelectric $T$ wave in that lead, a deep S wave in standard leads II and III with a positive T wave in those leads. He stated that a clear QI occurred also, and that in IVR there was a deep S (QS) wave and that the RS-T segment was somewhat elevated. Actually a few years earlier than this Sigler and Schneider (1935) were the first to point out that in apical aneurysms there was a tendency for the major QRS deflections to be deflected downwards in lead II and III. Apart from Nordenfelt's emphasis on the low $\mathbf{R}$ wave in standard lead I, which in our records is commonly tall, as well as in VL, we would agree that the QI, TI, SII, SIII appearance is in itself most suggestive of a cardiac aneurysm, although it is true that' this appearance may occur without aneurysm. Such deflections were seen in no fewer than 18 of our 24 proven anterior aneurysm cases, and if a QS wave is accepted instead of a Q wave then the figure of 18 rises to 20 . But these deflections were also seen in two of the nine cases of anterior infarct where no aneurysm was present at autopsy.

In the same year as Nordenfelt's paper, Eliaser and Konigsberg (1939) stated that the cardiograms obtained from cases of ventricular aneurysm could be considered as falling into four groups. Their first group showed what they termed left axis deviation, and corresponds fairly closely to the one mentioned by Nordenfelt. They thought that the cardiogram was not peculiar to cardiac aneurysm but occurred sufficiently often to warrant consideration of it. Their second group showed what they termed right axis deviation, with a large $\mathrm{S}$ wave in lead $\mathrm{I}$ accompanied by an inverted $\mathrm{T}$ wave in that lead. It can be seen from their illustrations that this so-called $S$ wave is in fact a QS wave and that the $R$ wave is absent. This apparent right axis deviation is really due to the fact that VL records the cavity potential from inside the left ventricle (QS) owing to the death of the intervening muscle tissue. As we have said, we encountered four such aneurysms where there was a QS wave in lead VL, and thus also in lead I, and none of the ten cases of anterior infarction without aneurysm showed such a QS wave. Rowland (1943) even states that when this " right axis deviation" appearance is present it is of greater diagnostic value than the TI, SII, SIII type of curve. Gross and Schwedel (1941) also conclude that the single positive finding in ventricular aneurysm is the comparative frequency of " right axis deviation." Recently Laham (1950) has stated that this appearance of right axis deviation is the commonest finding in ventricular aneurysm. We would agree that the absence of an $R$ wave in standard lead I with the production of a QS wave in that lead is in itself highly suggestive of the presence of an anterior aneurysm, but in our experience it is not the commonest cardiographic appearance to accompany ventricular aneurysm.

Dressler and Pfeiffer (1940) were the first to point out that the cardiographic changes once developed appeared to be very constant, and a deep S deflection in leads II and III was striking in half their cases. Boyd and Scherf (1942) were the first to draw attention to the fact that the elevation of the RS-T segment was such that one was inclined to assume that a recent infarct had occurred but that when these cases of aneurysm were followed the cardiogram was found to retain the same appearance for years.

Wilson et al. (1944) confirmed the observation of Boyd and Scherf and extended it to include unipolar chest leads. They could offer no explanation as to why this persistent RS-T segment displacement should be present and thought it was merely a chance association with aneurysm. Later, however, Wilson et al. (1947) held the opinion that this permanent RS-T segment displace- 
ment was associated with ventricular aneurysm more often than one might suspect. This may well be the case, for the important lesion is the large infarct. Myers et al. (1948) had previously pointed out that this tendency for the persistence of the RS-T segment elevation in cardiac aneurysm rendered it impossible to estimate the age of an infarct from the study of one tracing without other clinical details. They thought the abnormal RS-T segment and T wave probably represented cavity potential transmitted through the aneurysm without modification when the wall was completely replaced by fibrous tissue, or transmitted with slight alteration when the wall contained some remnants of muscle. They also pointed out that in addition to ventricular aneurysm causing segment shift very like a recent infarct, two other conditions could lead to similar changes, namely the occurrence of pericarditis independent of an old healed infarct, and the effect of cardiac glycoside superimposed on the curve due to an old healed infarct. The effect of digitalis on the RS-T segment and $T$ wave depend's upon the direction of the major phase of the QRS. In leads with a QS complex and upright $\mathrm{T}$ wave, full digitalization may result in further elevation of the RS-T segment and increased amplitude of the $T$ wave. Rosenberg and Messinger (1949) have recently pointed out that in cases with bundle branch block the persistence of the RS-T segment elevation in chest leads does not, of course, have the same significance as in cases where there is no block. Ford and Levine (1949) confirmed the opinion of Wilson and his co-workers that persistent elevation of the RS-T segment, although non-specific, should lead the clinician to suspect the presence of ventricular aneurysm; it was present in seven of their ten cases.

While agreeing that persistent elevation of the RS-T segment is suggestive of cardiac aneurysm, we feel that it has perhaps been over emphasized. There seems no general agreement as to how long the RS-T segment elevation can persist in the absence of cardiac aneurysm. There was no segment elevation in 6 of our 24 proven anterior aneurysms. However, if the segment elevation is very great, say more than $5 \mathrm{~mm}$. in some chest leads, and persists for a matter of several months or more, especially if associated with QS waves across the chest, then in our opinion this is very strong evidence indeed of cardiac aneurysm (see Cases 5 and 6, Fig. 9 and 10). The cause of this elevation of the RS-T segment remains obscure. The explanation of Moyer and Hiller (1951) that elevation of the RS-T segment that persists long after the stage of injury is caused by hypertrophy of the posterior wall of the heart opposite to the lesion, exerting its effect through dead intervening tissue, is attractive. Hypertrophy of the left ventricle was present in 7 of our 11 autopsy cases with aneurysm. The weight of the heart was recorded in five, namely $480,500,615,850$, and 885 grammes, and it is interesting to note that the greatest segment elevation was seen in the heaviest heart.

Goldberger and Schwartz (1948) claim that the QRS is upward in VR when ventricular aneurysm is present, and that the absence of this feature excludes the lesion. Our records do not entirely support this view, for in 7 of our 27 proven cases the $R$ wave in VR was tiny, being less than $1 \mathrm{~mm}$. and in one case it was absent altogether (Case 3, Fig. 5). But the fact that it may occur, perhaps from a change of position, fits in with the next point.

We consider that the S wave in VF, and consequently in standard leads II and III, is the important sign, for it determines the form of the bipolar limb leads. This deflection, more than $5 \mathrm{~mm}$. deep, was present in no fewer than 18 of our 24 cases of proven aneurysm. In all these where VF showed a deep $S$ wave, VL showed a tall $R$ wave (over $5 \mathrm{~mm}$.). This finding would show that the unipolar limb leads indicate an electrically horizontal heart when aneurysm results from anterior infarction.

\section{SUMMARY AND CONCLUSIONS}

If cardiographic appearances such as we have described are encountered, then provided there is no reason to suspect that the infarct is recent or that digitalis is affecting the cardiogram or that recent pericarditis has occurred, an anterior cardiac aneurysm is likely to be found at autopsy.

The four essential features are: (1) the presence of QS waves in one or more chest leads (ex- 
cluding VI); (2) inversion of the T wave, or its incorporation in the elevated RS-T segment, in at least three of the unipolar chest leads; (3) the presence of a deep S wave in lead VF, at least $5 \mathrm{~mm}$. in depth, and consequently in standard leads II and III also; (4) inversion of the T wave in VL and therefore also in standard lead I. Two other very common but not essential points are persistent elevation of the RS-T segment in unipolar chest leads and the presence of a $Q$ wave in lead VL and thus in standard lead $I$.

These were the findings in 18 out of 24 anterior aneurysms, and in a total of 20 if a QS wave instead of a $\mathrm{Q}$ wave is included in lead VL and standard lead I. Once these cardiographic appearances have developed they appear to be constant.

We do not, of course, claim that all anterior cardiac aneurysms produce this appearance and examples are quoted and illustrated where it is absent. However, when it is present this appearance is so highly suggestive, if not pathognomic, of cardiac aneurysm that it should lead to very careful fluoroscopic search for the presence of one. In three of our cases the ventricular aneurysm was unsuspected radiologically but more careful search as a result of the cardiographic appearance confirmed the presence of an aneurysm. In a fourth case, a large and persistent pleural transudate due to heart failure obscured the left border of the heart until death but the presence of a ventricular aneurysm was confidently diagnosed from the cardiogram and was subsequently confirmed at autopsy.

In spite of statements to the contrary, the presence of aneurysm is compatible with the absence of an $R$ wave in unipolar limb lead VR and one such case is illustrated.

We could find no cardiographic evidence diagnostic of the presence of a posterior cardiac aneurysm, including œesophageal leads in two cases, but in all three of our examples of posterior aneurysm the cardiogram showed that the damage had also extended round to involve the apex of the heart, and a QS wave was present in lead VF. A septal aneurysm was present in one anterior case but there was no cardiographic evidence to suggest this.

It might be thought that any large transmural antero-lateral infarct would give rise to an appearance such as we have described, but it was not found in ten such cases where aneurysm was excluded at autopsy, and this is in keeping with the results of other workers who have correlated closely the cardiogram and morbid anatomy of the infarct. Although the features of the chest leads can be the same in large anterior infarcts with or without aneurysmal bulging, when the limb leads are also included such an appearance as we have described as common in cardiac aneurysm is most unlikely to result from an old antero-Jateral infarct without the formation of an aneurysm.

The final conclusion of this study would be that as far as the chest leads are concerned, large anterior infarcts and aneurysms resulting from large anterior infarcts resemble one another, and this is what one would expect. There is no particular reason why a dead fibrotic ventricular wall that is thin enough to bulge and become an aneurysm should give rise to chest lead cardiograms peculiar to itself and distinct from a large transmural infarct that does not bulge. In either case the cardiograms may remain unchanged for years.

The cardiographic difference between aneurysmal and non-aneurysmal infarcts seems to be more in the limb leads. To some degree the interception of cavity potentials by lead VL will have its effect here, and so cause the important and characteristic QS in standard lead I. But apart from this the question of the electrical position. of the heart arises. We think that for some reason the presence of a ventricular aneurysm seems to affect this position and causes a deep $S$ wave in VF which appears also in standard leads II and III.

To understand the reason for certain cardiographic appearances is interesting and useful. But when they help the diagnosis of ventricular aneurysm we wonder whether it is important. These lesions do not seem to carry any worse prognosis than other large infarcts. We regard them as more curious than lethal. The ultimate outlook for the patient depends on the fate of the muscle that survives, not on the loss of the muscle that has died. 


\section{REFERENCES}

Baillie, M. (1793). Morbid Anatomy. 1st ed., London.

Boyd, L. J., and Scherf, D. (1942). Med. Clin. N. Amer., 26, 919.

Dressler, W., and Pfeiffer, R. (1940). Ann. intern. Med., 14, 100.

Eliaser, M., and Konigsberg, J. (1939). Arch intern. Med., 64, 493.

Ford, R. V., and Levine, H. D. (1949). Scientific Session Amer. Heart Assoc., p. 24.

Goldberger, E., and Schwartz, S. P. (1948). Amer. J. Med., 4, 243.

Gross, H., and Schwedel, J. B. (1941). New York State J. Med., 41, 488.

Hochrein, M. (1932). Der Coronarkreislauf. Julius Springer, Berlin.

Laham, J. (1950). La France Médicale, 10, 1.

Moyer, J. B., and Hiller, G. I. (1951). Amer. Heart J., 41, 340.

Myers, G. B., Klein, H. A., and Hiratzka, T. (1948). Amer. Heart J., 36, 838.

Nordenfelt, O. (1939). Acta. med. Scandinav., 102, 101.

Parkinson, J.; Bedford, D. E., and Thomson, W. A. R. (1938). Quart. J. Med., 7, 455.

Rosenberg, B., and Messinger, W. J. (1949). Amer. Heart J., 37, 267.

Rowland, D. (1943). Ann. intern. Med., 19, 349.

Samet, P., Schwedel, J. B., and Mednick, H. (1950). Amer. Heart J., 39, 749.

Schwedel, J. B. (1948). Clinical Roentgenology of the Heart. New York.

Sigler, L., and Schneider, J. J. (1935). Ann. intern. Med., 8, 1033.

Wilson, F. N., Johnston, F. D., Rosenbaum, F. F., Erlanger, H., Kossman, C. E., Hecht, H., Cotrim, N., de Oliveira, R. M., Scarsi, R., and Barker, P. S. (1944). Amer. Heart J., $27,19$.

-, Rosenbaum, F. F., and Johnston, F. D. (1947). Advances in Internal Medicine., 2, 1. Interscience Publishers, New York. 